\title{
Time variations of cosmic-ray helium isotopes with BESS-Polar I
}

\author{
K. Abe ${ }^{\text {a }}$, H. Fuke ${ }^{\text {b }}$, S. Haino ${ }^{\text {c }}$, T. Hams ${ }^{\text {d }}$, M. Hasegawa ${ }^{\text {c }}$, A. Horikoshi ${ }^{\text {c }}$, A. Itazaki ${ }^{\text {a }}$, \\ K.C. Kim ${ }^{\mathrm{e}}$, T. Kumazawa ${ }^{\mathrm{c}}$, A. Kusumoto ${ }^{\mathrm{a}}$, M.H. Lee ${ }^{\mathrm{e}}$, Y. Makida ${ }^{\mathrm{c}}$, S. Matsuda ${ }^{\mathrm{c}}$, \\ Y. Matsukawa $^{\mathrm{a}}$, K. Matsumoto ${ }^{\text {c }}$, J.W. Mitchell ${ }^{\mathrm{d}}$, A.A. Moiseev ${ }^{\mathrm{d}}$, J. Nishimura ${ }^{\mathrm{e}}$, \\ M. Nozaki ${ }^{c}$, R. Orito ${ }^{a}$, J.F. Ormes ${ }^{\mathrm{f}}$, N. Picot-Clémente ${ }^{\mathrm{e}, *}$, K. Sakai ${ }^{\mathrm{d}}$, M. Sasaki ${ }^{\mathrm{d}}$ \\ E.S. Seo ${ }^{\text {e }}$, Y. Shikaze ${ }^{\text {a }}$, R. Shinoda ${ }^{\mathrm{g}}$, R.E. Streitmatter ${ }^{\mathrm{d}}$, J. Suzuki ${ }^{\mathrm{c}}$, Y. Takasugi ${ }^{\mathrm{a}}$ \\ K. Takeuchi ${ }^{\mathrm{a}}$, K. Tanaka ${ }^{\mathrm{c}}$, N. Thakur ${ }^{\mathrm{f}}$, T. Yamagami $^{\mathrm{c}}$, A. Yamamoto $^{\mathrm{c}}$, T. Yoshida $^{\mathrm{b}}$ \\ K. Yoshimura ${ }^{\mathrm{c}}$ \\ ${ }^{a}$ Kobe University, Kobe, Hyogo 657-8501, Japan \\ ${ }^{\mathrm{b}}$ Institute of Space and Astronautical Science, Japan Aerospace Exploration Agency (ISAS/JAXA), Sagamihara, Kanagawa 252-5210, Japan \\ ${ }^{\mathrm{c}}$ High Energy Accelerator Research Organization (KEK), Tsukuba, Ibaraki 305-0801, Japan \\ ${ }^{\mathrm{d}}$ National Aeronautics and Space Administration, Goddard Space Flight Center (NASA/GFSC), Greenbelt, MD 20771, USA \\ ${ }^{\mathrm{e}}$ Institute for Physical Science and Technology (IPST), University of Maryland, College Park, MD 20742, USA \\ ${ }^{\mathrm{f}}$ University of Denver, Denver, CO 80208, USA \\ ${ }^{\mathrm{g}}$ The University of Tokyo, Bunkyo, Tokyo 113-0033, Japan
}

Available online 2 October 2013

\begin{abstract}
The Balloon-borne Experiment with a Superconducting Spectrometer (BESS) is configured with a solenoidal superconducting magnet and a suite of precision particle detectors, including time-of-flight hodoscopes based on plastic scintillators, a silica-aerogel Cherenkov detector, and a high resolution tracking system with a central jet-type drift chamber. The charges of incident particles are determined from energy losses in the scintillators. Their magnetic rigidities (momentum/charge) are measured by reconstructing each particle trajectory in the magnetic field, and their velocities are obtained by using the time-of-flight system. Together, these measurements can accurately identify helium isotopes among the incoming cosmic-ray helium nuclei up to energies in the GeV per nucleon region. The BESS-Polar I instrument flew for 8.5 days over Antarctica from December 13th to December 21st, 2004. Its long-duration flight and large geometric acceptance allow the time variations of isotopic fluxes to be studied for the first time. The time variations of helium isotope fluxes are presented here for rigidities from 1.2 to $2.5 \mathrm{GV}$ and results are compared to previously reported proton data and neutron monitor data.
\end{abstract}

Published by Elsevier Ltd. on behalf of COSPAR.

Keywords: Cosmic-ray isotopes; Cosmic-ray flux time variations; Solar modulation; Solar activity

\section{Introduction}

The central goals of the BESS program are to precisely measure low energy cosmic-ray antiprotons (Abe et al., 2012) and to search for antihelium in the cosmic rays (Abe et al., 2012). In addition, the BESS instruments allow

\footnotetext{
* Corresponding author. Tel.: +1 2025097831.

E-mail address: picot@umd.edu (N. Picot-Clémente).
}

fluxes of hydrogen and helium isotopes to be determined (Wang et al., 2002). The measurements of the fluxes of antiprotons and normal nuclei give complementary information on solar modulation and provide information on cosmic-ray origins and propagation in interstellar space.

BESS (Yamamoto et al., 2013) is a Japanese-US program that has carried out 11 successful balloon flights, 9 of which took place in northern Canada from 1993 to 2002. To take advantage of low geomagnetic cutoff and 


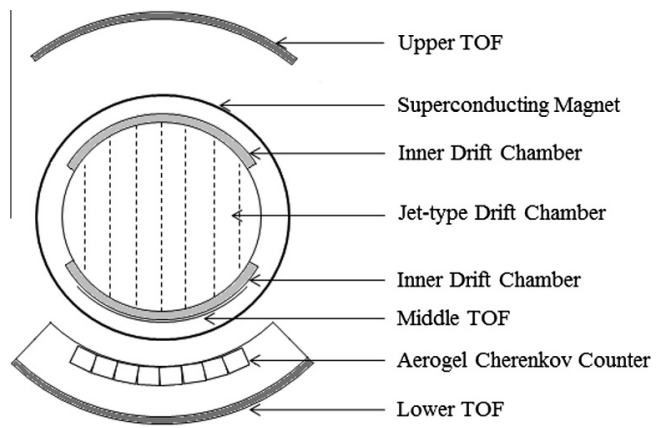

Fig. 1. Cross-sectional view of the BESS-Polar I spectrometer.

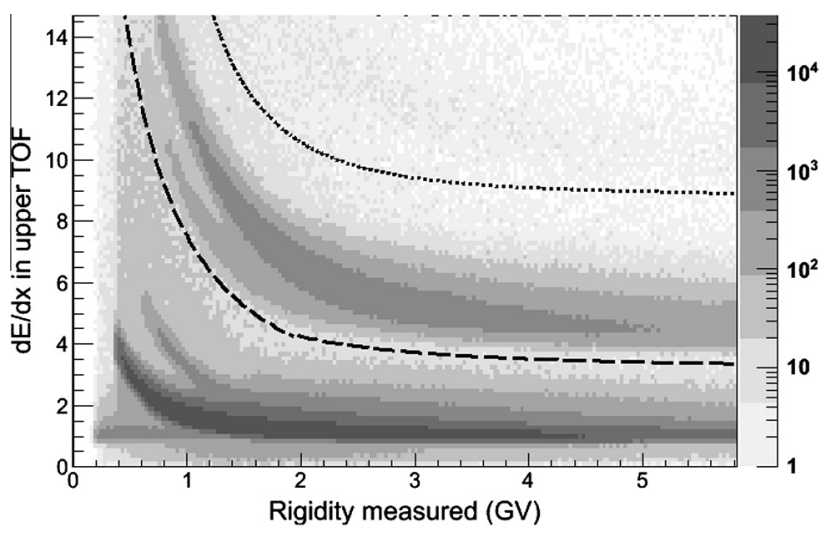

Fig. 2. The $d E / d x$ in the upper ToF counter versus the measured rigidity for the whole BESS-Polar I flight data set. The long-dashed line represents the cut selection used to separate $Z=1$ (below) and $Z=2$ (above) events with the upper ToF. The dotted line corresponds to the maximum $d E / d x$ allowed for $Z=2$ events.

improve the exposure time for data taking the BESS-Polar I and BESS-Polar II instruments were developed for Antarctic long-duration balloon flights and were flown for 8.5 days in December 2004 and 24.5 days in December 2007/January 2008 respectively.

These long-duration flights over Antarctica allow studies of cosmic-ray flux time variations on time scales of a few hours, and a preliminary work on proton transient variations was reported in Thakur et al. (2011). With the BESS-Polar I instrument, time variations of ${ }^{3} \mathrm{He}$ and ${ }^{4} \mathrm{He}$
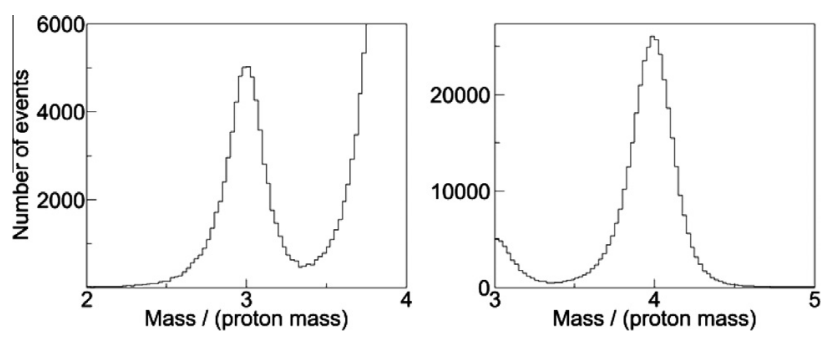

Fig. 3. Mass distributions of ${ }^{3} \mathrm{He}$ (left) and ${ }^{4} \mathrm{He}$ (right) events for a rigidity range from $\sim 1.2$ to $\sim 2.5 \mathrm{GV}$ for the whole flight data set. fluxes could be measured for the first time. They are presented in this paper from the full flight period of December 13th to December 21st 2004.

After presenting the instrument in Section 2, the analysis to separate isotopic elements is described in Section 3, and the time variations of helium isotope fluxes are discussed in Sections 4 and 5.

\section{The BESS-Polar I instrument}

The BESS-Polar I detectors and the solenoidal magnet were concentrically arranged. From the top to the bottom of the instrument, a downward-going particle passes through an "upper" plastic scintillator hodoscope, a solenoidal superconducting magnet producing a uniform magnetic field of $0.8 \mathrm{~T}$, two layers of inner drift chambers, a central jet-type drift chamber, a "middle" plastic scintillator hodoscope, an aerogel Cherenkov counter, and another "lower" plastic scintillator hodoscope. A cross-sectional view of the BESS-Polar I spectrometer is shown in Fig. 1. More detailed information can be found in Abe et al. (2008).

The difference in the measured times when a particle passes the top and bottom (or top and middle) layers of time-of-flight hodoscopes (ToF) gives the velocity of the particle. A time resolution of about $120 \mathrm{ps}$ is achieved for singly-charged particles, yielding a resolution of about $3 \%$ in the velocity $\beta$.

From the ionization energy losses $d E / d x$ in the ToF scintillators, the charge $Z$ of the particle is determined from:

$\frac{d E}{d x}=\left(\frac{Z}{\beta}\right)^{2} f(\beta)$.

Using hits in the ToFs and in the tracker, the gyroradius $\rho$ of the incoming particle in the magnetic field $B$ is measured by reconstructing its trajectory. The particle rigidity $R$ is then obtained from the relation:

$R=B \rho$

The mass $M$ of the crossing particle is then determined by:

$M=Z R \sqrt{1 / \beta^{2}-1}$.

The particle rigidity is measured inside the magnetic spectrometer. The corresponding kinetic energy per nucleon $E_{k}$ is:

$E_{k}=\sqrt{(R / A)^{2} Z^{2}+M_{p}^{2}}-M_{p}$,

where $M_{p}$ is the proton mass and $A$ the mass number of the particle. Its kinetic energy and rigidity at top of instrument are determined by correcting the measured energy for losses in the different layers in the upper part of the instrument. Based on Monte Carlo simulations (see Section 3), we estimate the rigidity resolution to be better than $1 \%$ below $5 \mathrm{GV}$ for both ${ }^{3} \mathrm{He}$ and ${ }^{4} \mathrm{He}$. 

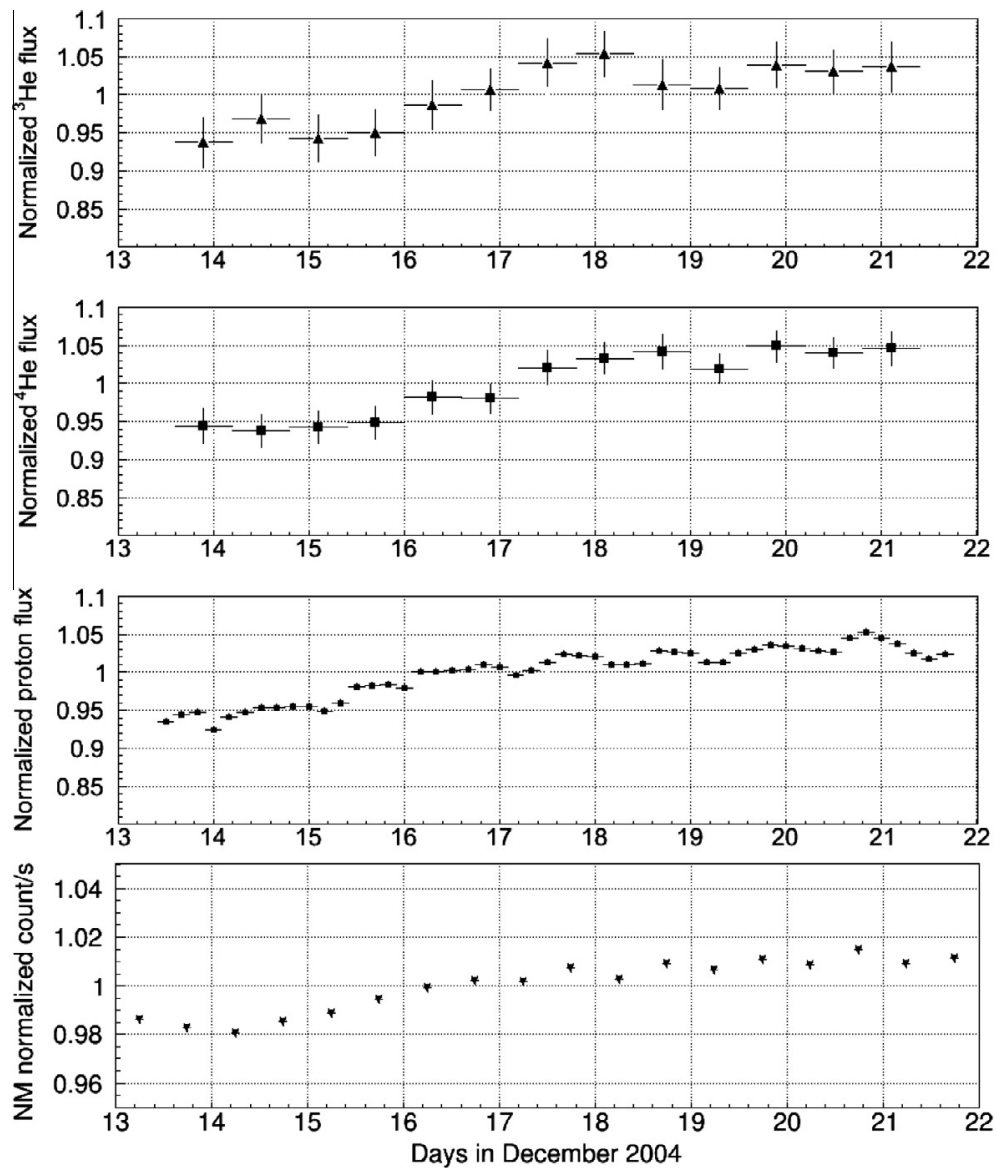

Fig. 4. From top to bottom are shown the flux time variations normalized by the mean flux values over the whole 13-21 December 2004 data taking period for ${ }^{3} \mathrm{He}$ and ${ }^{4} \mathrm{He}$ in the rigidity range from $\sim 1.2$ to $\sim 2.5 \mathrm{GV}$ with BESS-Polar I using a binning of time of $14.4 \mathrm{~h}$. The normalized proton flux in the rigidity range from $\sim 1.5$ to $\sim 2.1 \mathrm{GV}$ taken from Thakur et al. (2011) is also plotted, with a time resolution of $4 \mathrm{~h}$. The last plot shows the normalized counts per hour of the South Pole Neutron Monitor data (Bartol NM, 2004) averaged every $12 \mathrm{~h}$, sensitive to changes in cosmic-ray spectrum >5 GV.

\section{Data analysis to separate isotopes}

For the present study, helium isotopes crossing the BESS-Polar I spectrometer were simulated using the GEANT3 Monte Carlo code (Application Software Group, 1993). 800 million downward-going ${ }^{3} \mathrm{He}$ and ${ }^{4} \mathrm{He}$ particles were simulated incident isotropically on a semisphere above the instrument. Particles were selected from a flat energy spectrum $\left(d N / d E\right.$ proportional to $E^{-1}$ on a logarithmic scale) from 0.001 to $25 \mathrm{GeV}$. The GEANT3 simulations, which include a full set of hadronic interactions, are used primarily to determine the geometric acceptance of the detector and its detection efficiency, after rejecting events that interacted in the detector.

Due to a high failure rate when exposed simultaneously to low pressure and low temperature, only $60 \%$ of BESSPolar I ToF photomultipliers were operational during the flight. The analysis to separate isotopes requires precise measurements of velocity and charge. To achieve the best performance, only ToF scintillator paddles that had both photomultipliers working were used in the analysis. This led to a geometric acceptance of $0.05 \mathrm{~m}^{2} \mathrm{sr}$ at a rigidity of $2 \mathrm{GV}$.
As discussed above, the charge of the crossing particle can be determined from measurements of $d E / d x$ in the ToFs. To separate $Z=1$ and $Z=2$ events to identify hydrogen and helium, band cuts in $d E / d x$ vs. rigidity are used with data from both the upper and lower ToFs. To illustrate these cuts, Fig. 2 shows the selection cut applied to the $d E / d x$ vs. rigidity measured with flight data from the upper ToF. The distributions of ${ }^{1} \mathrm{H},{ }^{2} \mathrm{H},{ }^{3} \mathrm{He}$ and ${ }^{4} \mathrm{He}$ can be distinguished at low rigidities in this plot.

Many contaminating events are present in the data sample, and selection criteria are defined to remove these events. They are divided into two categories:

- The "single track" cuts remove particles interacting hadronically inside or above the detector. $d E / d x$ band cuts are among these selection criteria, and ensure that only $Z=1$ or $Z=2$ events are used in subsequent analysis. We require there to be only one hit each in the upper and lower ToFs, and only one reconstructed track. In addition to these selection cuts, it is required that the track pass through the center of the jet chamber to remove effects from the detector sides, and that the measured velocity is less than the speed of light to 

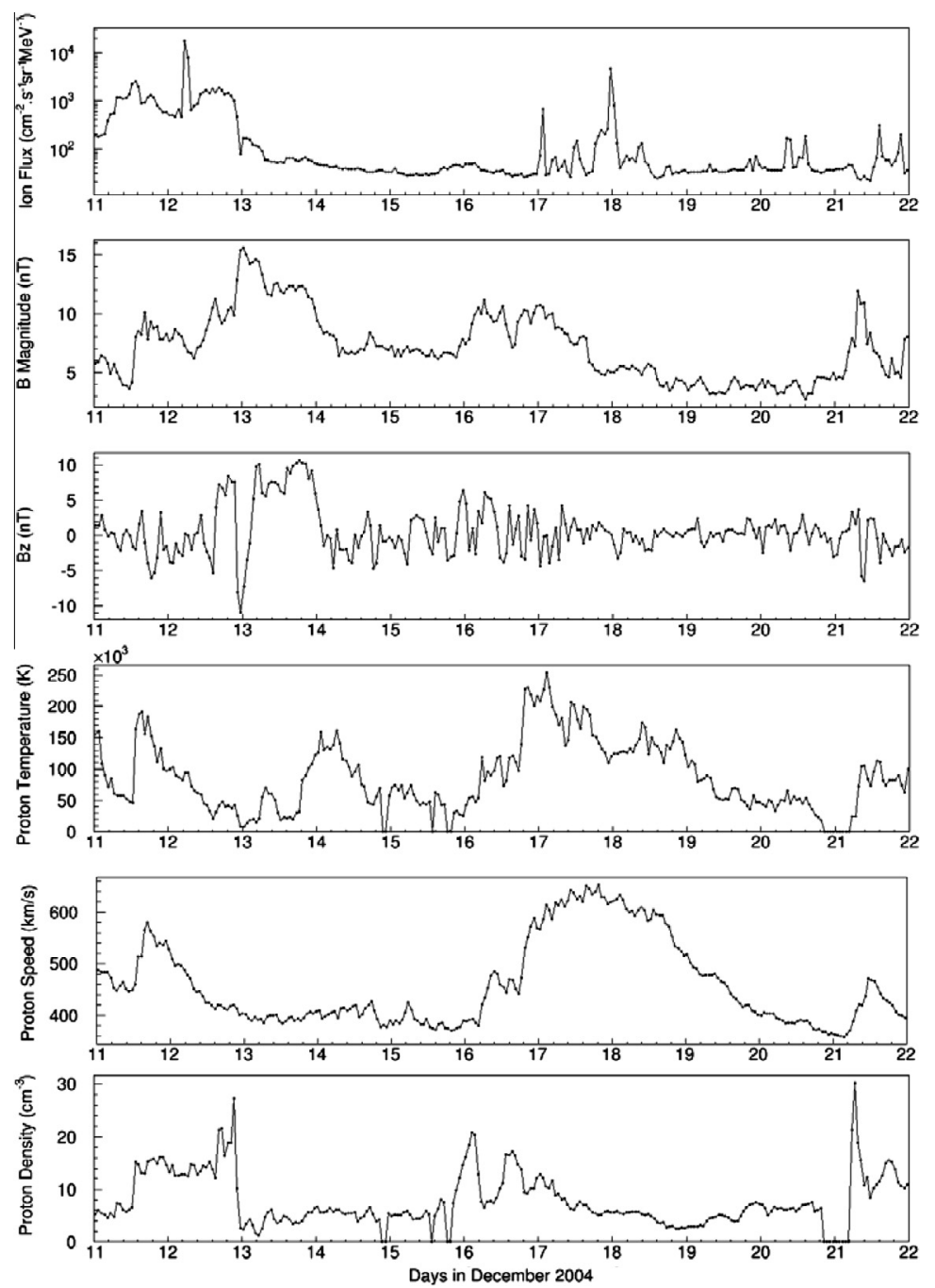

Fig. 5. Hourly ACE data (ACE, 2004) are presented in figure from 11 to 22 December. From top to bottom are shown the SEP flux (ion flux with energies from 0.066 to $0.114 \mathrm{MeV}$ ), the IMF magnitude, the IMF component in the $z$ direction, the proton temperature, the solar wind speed (proton speed) and the plasma density (proton density).

ensure real masses (c.f. Eq. (3)). The single-track cut selection efficiency is estimated with Monte Carlo simulations to be about $60 \%$.

- The "quality" cuts remove particles for which tracks are poorly reconstructed because of noise or detector limitations. To improve the quality of the data set, a minimum of 3 inner drift chamber pads and a maximum of 100 hits in the jet chamber are required. To get better measurements, only events with good fitting parameters $\chi^{2}$ in both $Z_{-}$ $Y$ and $r-\phi$ planes, and for which the measured rigidity has a good estimated error, are kept. Other criteria include consistency cuts that require a good match between the reconstructed track and hits in the tracking detectors and ToF paddles. Quality cuts give a selection efficiency of about $45 \%$.

After refining the flight data with the single-track and quality selection cuts, we determine particle masses from the measurements of rigidity, velocity and charge, as explained in Section 2. In order to separate ${ }^{3} \mathrm{He}$ and ${ }^{4} \mathrm{He}$, mass distributions are fitted by double Gaussian distributions. The numbers of remaining helium isotope events are then obtained from the integrals of the fitted Gaussian distributions. Fig. 3 shows the mass distributions of ${ }^{3} \mathrm{He}$ and ${ }^{4} \mathrm{He}$ events in the rigidity range from $\sim 1.2$ to $\sim 2.5 \mathrm{GV}$ for the whole flight data set. $1.2 \mathrm{GV}$ corresponds to the minimum rigidity needed for ${ }^{4} \mathrm{He}$ particles to cross both the atmosphere and the BESS-Polar I detector.

\section{Results}

Data collected by BESS-Polar I over the 8.5 -day period provide, for the first time, the possibility to measure flux time variations of light cosmic-ray isotopes at Earth. In this study, we calculate average fluxes for every $14.4 \mathrm{~h}$ of data, chosen to provide sufficient statistical precision to show the time-dependent variations in the measured helium isotope fluxes. The resulting statistical uncertainties are about $2.5 \%$ for ${ }^{3} \mathrm{He}$ and $1.5 \%$ for ${ }^{4} \mathrm{He}$. The calculation takes into 
account the time dependence of the instrument response, which is corrected by considering variations of efficiencies with time. The average atmospheric overburden of $4.3 \mathrm{~g} \mathrm{~cm}^{-2}$ during the flight is used to correct for particle energy losses in the atmosphere. Short-term variations in the atmospheric overburden during the flight, from 3.8 to $5.3 \mathrm{~g} \mathrm{~cm}^{-2}$, are not taken into account in this analysis. Their impacts on the measured isotope fluxes are negligeable compared to statistical uncertainties.

In Fig. 4 we show the normalized flux as a function of time for ${ }^{3} \mathrm{He}$ and ${ }^{4} \mathrm{He}$ during the BESS-Polar I flight using the mean time of each bin. The rigidity range from 1.2 to $2.5 \mathrm{GV}$ and data from 13 to 21 December 2004 were used. Distributions were normalized for every time bin for each isotope by dividing the average flux in that bin by the mean flux over the whole data taking period. For comparison, the previously reported transient variations of proton fluxes for rigidities from 1.5 to $2.1 \mathrm{GV}$ (Thakur et al., 2011) are displayed with a time resolution of $4 \mathrm{~h}$ and average statistical errors lower than $0.3 \%$. South Pole Neutron Monitor data (Bartol NM, 2004) are also shown using the normalized counts per hour averaged every $12 \mathrm{~h}$. The neutron monitor performs an integral spectral measurement of cosmic rays and is sensitive to changes in spectrum $>5 \mathrm{GV}$.

The time variations of helium isotope fluxes and previously reported proton transient variations, exhibit the same general behavior with a flux increase of about $10 \%$ from 13 to 17 December, followed by a quasi-stable period from 18 to 21 December. A similar tendency is observed in neutron monitor data, which shows a flux increase of about $3 \%$, followed by a more stable period.

\section{Discussion and conclusion}

Fig. 4 shows that fluxes of cosmic-ray ${ }^{3} \mathrm{He},{ }^{4} \mathrm{He}$ and protons, studied in similar rigidity ranges, varied similarly during the data taking period. The same physical processes must have caused the features observed in these time variations, and are most likely related to the changes in solar activity that occurred during the flight.

Sudden flux increases of about $10 \%$ are observed for cosmic-ray helium isotopes and for protons from 13 to 17 December, whereas neutron monitor data exhibit an increase of only about 3\%. Fluxes are shown for rigidities from 1.2 and $1.5 \mathrm{GV}$ respectively for helium isotopes and protons, and effectively for cosmic rays above $5 \mathrm{GV}$ from the South Pole Neutron Monitor. The differences in the relative increases in flux for different species and rigidity ranges are consistent with changes of solar activity that should affect low-rigidity particles more strongly.

Some interpretations can be proposed to explain the features observed in the flux time variations, as suggested in Thakur et al. (2011). The increase observed in the cosmic-ray fluxes between 13 to 17 December is most likely a recovery period after a Forbush decrease that would have occurred around 12 December. Forbush decreases are rapid decreases in the Galactic cosmic-ray intensity at
Earth followed by a gradual recovery period (Forbush, 1937; Lockwood, 1971). They are typically caused by transient solar activity such as a solar flare or coronal mass ejection. Around 12 December, ACE data (ACE, 2004), presented Fig. 5, show a flux increase of solar energetic particles (SEPs) matching the flux decrease observed in neutron monitor data. Such an event contains much lower energy particles than BESS-Polar I is designed to measure (Reames, 2004), however it has been observed that SEPs associated with a magnetic cloud (Burlaga, 1995) or corrotating interaction region (CIR) (Richardson, 2004) can cause transient variations in the Galactic cosmic rays. In Fig. 5, ACE data reveal a possible magnetic cloud around 12-13 December, characterized by a strength enhancement of the interplanetary magnetic field (IMF) with respect to its ambient value, by a large rotation of the IMF vector and by a proton temperature lower than average. In addition, the increase of solar wind speed and proton temperature around 12 December, along with a sharp decrease in plasma density around 13 December indicates the presence of a CIR. The possible magnetic cloud, CIR or the combination of both phenomena could have produced the small Forbush decrease between 12 to 13 December, which was followed by a flux recovery period from 13 to 17 December, observed with BESS-Polar I.

After 17 December, there was a stabilization of the plasma density leading to a quieter geomagnetic activity period, and making the observation of diurnal variations easier (c.f. Fig. 4 with proton and neutron monitor data) likely due to the motion of Earth through the isotropic Galactic cosmic rays (Compton and Getting, 1935).

\section{Acknowledgments}

The BESS-Polar experiment is a Japan-U.S. collaboration, supported by KAKEN-Hi (13001004 and 18104006) in Japan, and by NASA in the US. The authors thank NASA Headquarters, the NASA Balloon Program Office at Goddard Space Flight Center/Wallops Flight Facility, and the NASA Columbia Scientific Balloon Facility, as well as ISAS/JAXA and KEK for their support. Thanks to the US National Science Foundation (NSF) and Raytheon Polar Services Company for their professional support in the USA and in Antarctica. The authors thank the Bartol Research Institute neutron monitor program, supported by the NSF (Grant ATM-0000315), for providing their data. The authors thank the ACE SWEPAM, MAG, EPAM, and SIS instrument teams and the ACE Science Center for providing the ACE data.

\section{References}

Abe, K., Fuke, H., Haino, S., et al., 2008. Measurement of the cosmic-ray low-energy antiproton spectrum with the first BESS-polar antarctic flight. Phys. Lett. B 670, 103.

Abe, K., Fuke, H., Haino, S., 2012. Measurement of the cosmic-ray antiproton spectrum at solar minimum with a long-duration balloon flight over Antarctica. Phys. Rev. Lett. 108, 051102. 
Abe, K., Fuke, H., Haino, S., 2012. Search for antihelium with the BESSpolar spectrometer. Phys. Rev. Lett. 108, 131301.

Advanced Composition Explorer (ACE), December 2004 data. <http:// www.srl.caltech.edu/ACE/>.

Application Software Group, GEANT, Detector description and simulation tool, CERN program library long writeup W5013, 1993. <http:// wwwasd.web.cern.ch/wwwasd/geant/>.

Bartol research institute neutron monitor program, University of Delaware, December 2004 data. <http://neutronm.bartol.udel.edu/>.

Burlaga, L., 1995. Interplanetary Magnetohydrodynamics. Oxford University Press, New York.

Compton, A.H., Getting, I.A., 1935. An apparent effect of galactic rotation on the intensity of cosmic rays. Phys. Rev. 47, 817-821.

Forbush, S.E., 1937. On the effects in the cosmic-ray intensity observed during the recent magnetic storm. Phys. Rev. 51, 1108-1109.
Lockwood, J., 1971. Forbush decreases in the cosmic radiation. Space Sci. Rev. 12, 658-6715.

Reames, D.V., 2004. Solar energetic particle variations. Adv. Space Res. 34, 381-390.

Richardson, I.G., 2004. Energetic particles and corotating interaction regions in the solar wind. Space Sci. Rev. 111, 267-376.

Thakur, N., Abe, K., Fuke, H., et al., 2011. Transient variations in cosmic ray proton fluxes from BESS-Polar I and their physical interpretations. In: Proc. of the 32nd ICRC, abstract ID 1247.

Wang, J.Z., Seo, E.S., Anraku, K., et al., 2002. Measurement of cosmicray hydrogen, helium, and their isotopic composition with the BESS experiment. Astrophys. J. 564, 244-259.

Yamamoto, A., Mitchell, J., Yoshimura, K., et al., 2013. Search for cosmic-ray antiproton origins and for cosmological antimatter with BESS. Adv. Space Res. 51, 227-233. 